\title{
Review
}

Pharmacology

Pharmacology 2017;100:161-171

DOI: $10.1159 / 000477853$
Received: May 30, 2017

Accepted May 30, 2017

Published online: June 23, 2017

\section{Hyperemesis Gravidarum: A Review of Recent Literature}

\author{
Viktoriya London Stephanie Grube David M. Sherer Ovadia Abulafia \\ Division of Maternal-Fetal Medicine, Department of Obstetrics and Gynecology, State University of New York \\ (SUNY), Downstate Medical Center, Brooklyn, NY, USA
}

\section{Keywords}

Hyperemesis gravidarum · Nausea and vomiting of pregnancy $\cdot$ Therapeutic modalities

\begin{abstract}
Background: In the United States, hyperemesis gravidarum is the most common cause of hospitalization during the first half of pregnancy and is second only to preterm labor for hospitalizations in pregnancy overall. In approximately $0.3-$ $3 \%$ of pregnancies, hyperemesis gravidarum is prevalent and this percentage varies on account of different diagnostic criteria and ethnic variation in study populations. Despite extensive research in this field, the mechanism of the disease is largely unknown. Although cases of mortality are rare, hyperemesis gravidarum has been associated with both maternal and fetal morbidity. The current mainstay of treatment relies heavily on supportive measures until improvement of symptoms as part of the natural course of hyperemesis gravidarum, which occurs with progression of gestational age. However, studies have reported that severe, refractory disease manifestations have led to serious adverse outcomes and to termination of pregnancies. Summary: Despite extensive research in the field, the pathogenesis of hypereme-
\end{abstract}

sis gravidarum remains unknown. Recent literature points to a genetic predisposition in addition to previously studied factors such as infectious, psychiatric, and hormonal contributions. Maternal morbidity is common and includes psychological effects, financial burden, clinical complications from nutritional deficiencies, gastrointestinal trauma, and in rare cases, neurological damage. The effect of hyperemesis gravidarum on neonatal health is still debated in literature with conflicting results regarding outcomes of birth weight and prematurity. Available therapy options remain largely unchanged in the past several decades and focus on parenteral antiemetic medications, electrolyte repletion, and nutritional support. Most studies of therapeutic options do not consist of randomized control studies and cross-study analysis is difficult due to considerable variation of diagnostic criteria. Key Messages: Hyperemesis gravidarum carries a significant burden on maternal health and US health care. Most published research on pathogenesis is observational and suggests multifactorial associations with hyperemesis gravidarum. Precise, strictly defined criteria for clinical diagnosis are likely to benefit meta-analyses of further research studies regarding pathogenesis as well as therapeutic options.

(c) 2017 S. Karger AG, Basel

\section{KARGER}

(c) 2017 S. Karger AG, Basel

E-Mail karger@karger.com

www.karger.com/pha
David M. Sherer, MD

Division of Maternal-Fetal Medicine, Department of Obstetrics and Gynecology, State University of New York (SUNY), Downstate Medical Center, 450 Clarkson Avenue, Box 24, Brooklyn, NY 11203 (USA)

E-Mail davidmsherer@gmail.com 


\section{Introduction}

The origins of "hyperemesis gravidarum" are difficult to trace, as the term and the understanding of the disease process have evolved greatly throughout medical history. Although reports of maternal death from symptoms that now appear attributed to hyperemesis date as far back as religious documentation, historical medical literature cite Antoine Dubois, a consultant surgeon and a head obstetrician to Napoleon Bonaparte and his second wife Empress Marie Louise, as a physician who first identified the condition in 1852. Dubois is thought to have first described the syndrome during his address before the French Academy of Medicine when he spoke about the finding of "pernicious vomiting of pregnancy" [1]. One of the first written original accounts of the term was in 1897, when C.S. Bacon, an American physician, credited the term of "hyperemesis gravidarum" and its "3-stage" classification to Dubois. This literature marks a milestone of a change in the outlook upon this condition as a medical complication rather than a normal aspect of pregnancy. After the original description, the literature of late 19th and early 20th centuries flourished with suggestions of possible etiologies of disease including hypothesis such as "irritation of the vomiting reflex from the stretching of the uterine fibers," and "irritation of the cervix." "Toxinemia" was named as another possibility and was interestingly cited in association with hyperemesis gravidarum as well as eclampsia and "hysteria" $[2,3]$. The etiology of hyperemesis gravidarum has not been fully elucidated and this is currently believed to reflect a multifactorial disease process.

\section{Epidemiology}

The prevalence of hyperemesis gravidarum is approximately $0.3-3 \%$ of pregnancies and varies on account of different diagnostic criteria and ethnic variation in study populations. Notwithstanding, most studies agree that hyperemesis gravidarum is more common among young, primiparous mothers who are non-Caucasian and nonsmokers $[4,5]$. Within the US population, there is lack of significant data on the difference in prevalence of hyperemesis gravidarum between different ethnic backgrounds. Worldwide, women of Asian and Middle Eastern ethnicities have been reported to have higher rates of prevalence, even as high as approximately $10 \%$ in a study reported from a Chinese population [6]. It is important, however, to note that due to the lack of uniform diagnos- tic criteria, these percentages may be higher when diagnosis of a more mild form of nausea and vomiting are included.

\section{Clinical Diagnosis}

According to the latest American College of Obstetricians and Gynecologists (ACOG) guidelines on Nausea and Vomiting during pregnancy (2015), there still is no single accepted definition of hyperemesis gravidarum. The most commonly cited criteria for diagnosis of hyperemesis gravidarum include persistent vomiting not related to other causes, an objective measure of acute starvation (usually large ketonuria on urine analysis), electrolyte abnormalities and acid-base disturbances, as well as weight loss. Weight loss is often cited as at least 5\% loss of pre-pregnancy weight [4]. Serum electrolyte and acidbase abnormalities may include hypochloremic alkylosis, hypokalemia, and hyponatremia [7]. Other abnormalities such as mild elevation in amylase, lipase, and liver function enzymes are also associated with hyperemesis gravidarum [8]. Hyperemesis gravidarum may also present with signs and symptoms associated with severe dehydration including orthostatic hypotension, tachycardia, dry skin, mood changes, and lethargy.

Recently, a classification system was created to categorize hyperemesis gravidarum called the PUQE (pregnancy-unique quantification of emesis and nausea) scoring index. This index accounts for the daily number of vomiting episodes, the length of nausea per day in hours, and the number of retching episodes per day [9]. Clinical research and medical practice have been yet to adopt a universal system of hyperemesis gravidarum classification the clinical diagnosis of exclusion, remains [4].

The lack of specific diagnostic criteria is one aspect of hyperemesis gravidarum, which makes it difficult to cross analyze clinical research studies. In this review of the topic, we attempt to differentiate between studies on nausea and vomiting of pregnancy versus studies of the severe form, hyperemesis gravidarum.

The differential diagnosis of patients with hyperemesis gravidarum is wide and includes infections, metabolic, gastrointestinal, neurologic, and iatrogenic causes [10]. Common diagnoses such as gastroenteritis, cholecystitis, hepatitis and biliary tract diseases, drug abuse/misuse, migraine headaches as well as more rare causes such as diabetic ketoacidosis, intracranial lesions leading to increased intracranial pressure and intestinal obstruction, should also be considered. Hyperemesis gravidarum is of- 
ten associated with failure to respond to outpatient management and often requires hospitalization.

The mainstay of the clinical work up for patients with hyperemesis gravidarum is a detailed evaluation to exclude other potential causes of presenting symptoms. The following laboratory tests are most commonly included in the initial assessment: complete blood count and serum metabolic panels, urinalysis for ketones and specific gravity, thyroid function studies, amylase/lipase levels and in early pregnancies, a serum beta human chorionic gonadotropin (hCG) level for evaluation of possible molar or multiple gestations. Notwithstanding, only a limited subset of these tests is used in a clinical day-to-day practice for a patient with an initial presentation with hyperemesis gravidarum. Of note, the ACOG 2015 guidelines recommend that serum thyroid function studies should be obtained only in the presence of other signs of hyperthyroidism such as a palpable goiter. Several studies also recommend testing for Helicobacter pylori infection, as gastric ulcers can be a contributing factor to persistent, refractory hyperemesis gravidarum [4].

\section{Etiology}

Since our earlier review of this topic in 2000 , we note limited advancement in the understanding of the precise etiology of hyperemesis gravidarum [11]. No discrete mechanism of pathogenesis has yet been established, but the number of proposed associations continues to suggest that the etiology of hyperemesis gravidarum is likely multifactorial. A recent comprehensive literature meta-analysis, attempted to combine the evidence of all available studies of markers of hyperemesis gravidarum to evaluate for a possibility of an unequivocal test for this condition. For purposes of this study, the authors defined hyperemesis gravidarum as any combination of nausea, vomiting, dehydration, weight loss, or hospitalization for nausea, and/or vomiting in pregnancy in the absence of any other obvious cause for these complaints.

After thorough evaluation, the authors selected 81 publications, which met the desired criteria (such as casecontrolled population) for final analysis. They reported that $65 \%$ of cases had a marker of ketonuria; however, ketonuria did not appear to correlate with disease severity. Meta-analysis, with the use of the hierarchical summary receiver operating characteristics model, yielded an odds ratio of 3.2 (95\% CI 2.0-5.1) of H. pylori for hyperemesis gravidarum, as compared with asymptomatic control subjects (sensitivity, 73\%; specificity, 55\%). Stud-

Hyperemesis Gravidarum ies on hCG and thyroid hormones, leptin, estradiol, progesterone, and white blood count showed inconsistent associations with hyperemesis gravidarum; lymphocytes tended to be higher in women with hyperemesis gravidarum [12]. The following sections present a review of the proposed etiologies factors of hyperemesis gravidarum.

\section{Psychiatric Background}

Psychiatric history as an etiology of hyperemesis gravidarum is mostly considered to be a historic perspective; however, a number of current studies of this historically postulated association have been recently published. The largest of these studies to date, a Norwegian study published in 2017, assessed the association between depression and hyperemesis gravidarum and noted that the lifetime history of depression was associated with higher ORs for developing hyperemesis gravidarum (OR 1.49, 95\% CI 1.23-1.79) [13]. However, this study also noted that two thirds of women with hyperemesis gravidarum had neither a history of depression nor any symptoms of postpartum depression, and only $1.2 \%$ of women with a history of depression developed hyperemesis gravidarum. Given that only $1.2 \%$ of women with a history of depression developed hyperemesis gravidarum, and that the majority of women with hyperemesis gravidarum had no symptoms of depression, the study's authors concluded that depression not a likely etiology of hyperemesis gravidarum [13]. Current studies regarding this topic are targeted at evaluating the development of depression, anxiety, posttraumatic stress disorder, and other psychiatric disorders as an effect of hyperemesis gravidarum, rather than a cause [14].

\section{Hormonal Aspects}

A number of hypotheses regarding hormonal causes of hyperemesis gravidarum exist; yet there are no published studies to date that support a definitive causal relationship. Levels of serum hCG and progesterone, both produced by the placenta and corpus luteum during the firsttrimester of pregnancy, are temporally related to the symptoms of hyperemesis gravidarum, and have been implicated in pathophysiology of this condition. Researchers have also investigated an association between estrogen levels and hyperemesis.

\section{Human Chorionic Gonadotropin}

Available studies do not show a direct correlation between serum hCG levels and hyperemesis; however, they do demonstrate a correlation between hyperemesis and conditions of increased hCG such as multiple gestations 
and molar pregnancies. A meta-analysis of published studies investigating the correlation between increased serum levels of hCG and hyperemesis gravidarum between 1966 and 2005, noted that out of 18 published studies, 11 showed a positive association. Notwithstanding, these studies demonstrate correlation, but none of these studies confirmed a possible mechanism or causal association [15].

\section{Estrogen}

Estrogen has been linked to hyperemesis gravidarum mainly due to the association between women with hyperemesis and conditions known to have elevated levels of estrogen, such as obesity. Symptoms of nausea and vomiting in women with elevated levels of estrogen in the setting of oral contraceptive use also support this etiological hypothesis. One proposed mechanism of how estrogen results in symptoms of nausea and vomiting is due to the established fact that estrogen can decrease both gastric emptying and overall intestinal transit time. However, in the setting of hyperemesis gravidarum, recent gastrointestinal motility studies show that patients manifesting hyperemesis gravidarum have faster, not slower motility rates [15]. To date, no strong association between estrogen levels and hyperemesis gravidarum has been published.

\section{Progesterone}

Studies have examined a possible correlation between progesterone and onset of hyperemesis gravidarum due to a hypothesis that progesterone alone, or in combination with estrogen, may cause gastric dysrhythmias by decreasing gastric smooth muscles contractility [10]. Although research has shown that progesterone levels peak during the first trimester of pregnancy, no association with hyperemesis gravidarum has been established.

\section{Helicobacter pylori}

H. pylori, has long been implicated in the pathogenesis of hyperemesis gravidarum; however, only studies of association are available. For example, a correlation between $H$. pylori and the severity of nausea/vomiting of pregnancy (not specifically hyperemesis) has been demonstrated in a recent study in the Netherlands. This study of 5,549 women, 1,932 of whom, reported occasional vomiting and 601 of whom reported daily vomiting, demonstrated that women who were $H$. pylori positive were more likely to report daily vomiting with an adjusted odds ratio of 1.44. Additionally, women experiencing daily emesis and diagnosed with $H$. pylori infection had on average a $2.1 \mathrm{~kg}$ decrease in pregnancy weight gain and their infants had slightly reduced birth weight and increased risk of small for gestational age (SGA) status. In conclusion, the authors state that they believe H. pylori to be an independent risk factor for vomiting in pregnancy and suggested that future studies on eradication of H. pylori in pregnant women may be beneficial [16]. In meta-analysis showing the geographic association of H. pylori and hyperemesis gravidarum, most regions were noted to show a positive correlation. The differences in the strength of the correlation, however, suggest that H. pylori is unlikely to be the main mechanism in the pathophysiology of hyperemesis gravidarum. In North America, the OR of testing positive for $H$. pylori infection in the setting of hyperemesis gravidarum was found to be 2.33, in Europe OR 1.55, in Asia OR 3.27, Africa OR 12.38, and Oceania OR 10.93 [17]. Meta-analysis of case-control studies of $H$. pylori and hyperemesis gravidarum published in 2007 also demonstrated an overall positive correlation [18]. Although the data remain unclear regarding the role of $H$. pylori in the pathogenesis of hyperemesis gravidarum, ACOG 2015 guidelines advocate that $H$. pylori treatment is safe in pregnancy and could be beneficial in cases of refractory hyperemesis gravidarum.

\section{Genetics}

One of the more prevailing theories on the etiology of hyperemesis gravidarum is the importance of genetic factors in its pathogenesis. A 2008 study by Fejzo et al. [6] in California evaluated 1,224 patients with hyperemesis gravidarum and demonstrated the significance of family history in development of the disease. In this study, $28 \%$ of patients reported a history of hyperemesis gravidarum in their mother, 19\% reported a sister with hyperemesis gravidarum, and $9 \%$ of the patients reported at least 2 relatives with this condition. Among the most severe cases, those requiring total parenteral nutrition (TPN) or nasogastric feeding tube, the proportion of patients with affected sisters was even higher at $25 \%$. Given the low rates of hyperemesis gravidarum among the general population, the above associations give strong support to the theory of genetic predisposition for hyperemesis gravidarum.

\section{Maternal Complications}

\section{Nutritional Deficiencies}

Multiple nutrient deficiencies have been identified in the setting of hyperemesis gravidarum. Thiamine, or vitamin B1, is a water-soluble vitamin deficiency, which can result in the setting of the persistent vomiting such as 
in hyperemesis gravidarum. This deficiency may lead to a syndrome called Wernicke's encephalopathy. Patients may present with neurologic symptoms ranging from lethargy and confusion to hyporeflexia, ataxia, and oculomotor symptoms including nystagmus and ophthamoplagia. While death associated with hyperemesis is rare, deaths that have occurred are typically associated with Wernicke's encephalopathy. The severity of the complications of this condition, highlight the necessity for early diagnosis and treatment [19]. Most patients with severe disease continued to manifest subsequent impairments, with complete remission of symptoms occurring in only a minority of patients. Symptoms that ultimately resolved often required months to dissipate [20]. Even patients who required TPN due to their hyperemesis are at risk of thiamine deficiency. Case reports have identified patients receiving TPN without thiamine in the mixture resulting in iatrogenic Wernicke encephalopathy [21].

If Wernicke encephalopathy is suspected for a patient, MRI may be useful in diagnosis. MRI may also be useful in identifying other severe complications of hyperemesis, such as central pontine myelinolysis [22].

Clinically significant deficiencies of the fat-soluble vitamin, vitamin-K, have been reported. This deficiency has been linked to adverse effects such as neonatal hemorrhage, and case reports have noted the development of coagulopathy from vitamin $\mathrm{K}$ deficiency that contributed to intraperitoneal hemorrhage intraoperatively in a young woman with large myoma and small bowel obstruction who had been diagnosed with hyperemesis. This coagulopathy may lead to increased blood loss during procedures and surgeries required during pregnancy [23].

Serum electrolyte disturbances of patients with hyperemesis result in severe hypokalemia. Potassium abnormalities have been noted to increase the mortality associated with hyperemesis. Case reports have identified profound hypokalemia leading to rhabdomyolysis in the setting of hyperemesis gravidarum [24].

\section{Esophageal Injury}

Esophageal laceration associated with hematemesis, known as Mallory-Weiss syndrome, may result from the repetitive wrenching associated with hyperemesis. When this barotrauma causes rupture of the esophagus (Boerhaave syndrome), pneumomediastium may result (Hamman's syndrome). While some patients with this complication may tolerate conservative management, others may require surgical intervention. This complication may be suspected in patients presenting with subcutaneous emphysema on physical exam or imaging [25].

Hyperemesis Gravidarum

\section{Psychosocial Effects}

Studies have shown that the psychosocial burden of hyperemesis may be underestimated. In a study by Poursharif et al. [26], patients afflicted with hyperemesis gravidarum were more likely to report that their health care providers did not realize how ill they were. This study also showed that of the 808 women with hyperemesis studied, $15 \%$ had at least one termination due to their symptoms, and another $12 \%$ "almost" underwent termination of pregnancy. Many patients with hyperemesis reported fear of subsequent pregnancy due to their experience with hyperemesis. The patient-physician relationship may be strained by care giver attitude toward hyperemesis, as some have associated hyperemesis as a manifestation of psychosocial stressors or negative attitude toward that pregnancy [26]. A study from 2001 by Simpson et al. [27] utilized the Minnesota Multiphasic Personality Inventory-2 and Symptom Check List-90 Revised in order to compare patients with hyperemesis to controls and found no support that hyperemesis was solely a psychological manifestation [27].

Another study noted an association between hyperemesis gravidarum and posttraumatic stress syndrome, with women reporting episodes reexperiencing, avoidance/numbing, and hyperarousal. Women demonstrating these symptoms were found to experience difficulty with breast milk production, experience marital difficulties, work and education problems, financial difficulties, as well as poor self-care postpartum [14]. A study using the Beck depression inventory that compared 200 women with hyperemesis with 200 matched controls noted that the depression risk was more than 76 -fold in the group with hyperemesis [28].

In the United States, hyperemesis gravidarum is the most common cause of hospitalization during the first half of pregnancy and is second only to preterm labor for pregnancy overall [16]. Similar findings were also found to be true for other countries including Canada and many European countries. Although this disease is associated with many psychosocial challenges, a study by Tan et al. [29] found that while patients with hyperemesis experience significant distress in the first trimesters of pregnancy, significant rebound and improvement in results from Depression, Anxiety, and Stress scale in hospitalized patients were found. This suggests that although the distress is severe, a portion of the distress experienced with hyperemesis is self-limiting [30]. 


\section{Invasive Resuscitation}

Patients with hyperemesis gravidarum that are unable to tolerate oral intake sometimes require sustenance through TPN. Patients who require TPN for nutritional support are likely to require central line placement. Central line catheters have been associated with complications such as infections, thrombosis, hematomas, pneumothoracies, and cardiac arrhythmias [21].

A case series of 5 patients who required jejunostomy placement for nutrition administration, including $1 \mathrm{pa}-$ tient who had a jejuonstomy placed in 2 consecutive pregnancies, was considered. In this series, each pregnancy was carried to term. Minimal complications were reported, limited only to tube dislodgement, making jejunostomy a potentially safe option for patients with severe hyperemesis [31].

\section{Fetal Outcomes}

Research on fetal outcomes after in-utero exposure to maternal hyperemesis gravidarum presents conflicting outcomes. The main focus of research on hyperemesis gravidarum and associated fetal outcomes is centered on preterm birth and low birth weight in the setting of hyperemesis gravidarum. Recent examples of such studies include a Norwegian population study of over 150,000 patients, 1,200 of who had hyperemesis gravidarum. This study showed a positive association between adverse neonate outcomes and hyperemesis gravidarum. Compared with infants born to women without hyperemesis, rates of low birth weight and preterm delivery were $8 \%$ higher than rates among infants born to women with hyperemesis and low pregnancy weight gain. The study found no correlation between poor neonatal outcome and women with diagnosis of hyperemesis gravidarum who did not exhibit a less than 7-lbs pregnancy weight gain [32]. A Turkish study of pregnancies complicated by hyperemesis gravidarum between 2003 and 2011 showed no association between disease and the following primary neonatal outcomes: 5-min Apgar score of $<7$, low birth weight, SGA, preterm delivery, fetal sex, and stillbirth [33].

In addition, studies on hyperemesis gravidarum and abnormalities of "placental conditions" show conflicting effects. In 2016, Koudijs et al. [34] published on the relationship between hyperemesis gravidarum and placental disease. This study assessed 3 categories of correlations: (1) "placental insufficiency" disorders which included: gestational hypertension, preeclampsia, stillbirth, miscarriage; (2) "poor neonatal outcomes," which in- cluded birth weight, SGA, low birth weight, Apgar score at $5 \mathrm{~min}$, gestational age at delivery; and (3) "placental outcomes," which comprised of placental weight, and placental weight to birth weight ratio. The correlations were studied for positive or negative association with hyperemesis gravidarum and for with presence of correlation with severity of hyperemesis. Of all the above areas studied, the only positive association was a minimal difference (172 g) in birth weight between pregnancies exposed to severe hyperemesis gravidarum and their negative controls.

Conflicting with the above findings are the findings of a Swedish study published in 2013, which assessed over 1,000 patients with hyperemesis gravidarum. This study demonstrated a slight association between hyperemesis gravidarum in the first trimester and later occurring preeclampsia. This study also noted that women manifesting hyperemesis gravidarum with their first admission being in the second trimester had a more than twofold risk of preterm delivery ( $<37$ weeks) with preeclampsia, a threefold increased risk of placental abruption, and a 39\% increased risk of delivering an SGA infant [35].

Another positive association described between hyperemesis gravidarum and poor fetal outcomes is one of increased prevalence of psychiatric disorders, mainly anxiety, depression, and bipolar disorder, in adults who were exposed to hyperemesis gravidarum while in utero [36]. However, no other studies to date have been published in support or dispute of this data.

\section{Therapeutic Options}

The focus of the current research regarding hyperemesis gravidarum has mainly been in the field of treatment. A wide range of interventions have been studied in randomized control trials (RCTs) for management of hyperemesis gravidarum, starting from supportive measures, such as hydration, to preconception supplementation with prenatal vitamins for 3 months prior to conception. Various outpatient and inpatient pharmacological methods and alternative medicine methods such as acupuncture [4] have also been described.

The Cochran group performed a review of randomized clinical trials specifically targeting treatment for the true condition of hyperemesis gravidarum, attempting to separate these RCTs from treatment studies aimed at "nausea and vomiting" in pregnancy. The group reviewed 25 trials, involving approximately 2,052 women diagnosed with hyperemesis gravidarum, publishing their findings in 2016. It is important to note that by 
Cochran evidence standards, outcome data presented in most studies were graded as "low" or "very low" quality, mainly due to the "imprecision of effect estimates." This finding highlights the need for further research in the field and the importance of developing standard criteria of a diagnosis of hyperemesis gravidarum [5].

\section{Supportive Measures}

The mainstay of supportive measures is to provide adequate rehydration and electrolyte repletion. Current initial management recommendations include changing the patient to NPO status, providing immediate fluid resuscitation with boluses of normal saline or lactate ringers solution. Continuous hydration should then be maintained with a solution containing 5\% dextrose. Studies have shown faster improvement of nausea symptoms with dextrose-containing fluids [37]. Additionally, serum electrolytes especially magnesium, phosphate, potassium should be repleted and sodium levels should be monitored. To prevent Wernicke's encephalopathy, $100 \mathrm{mg}$ of thymine should be administered at initiation of rehydration [38]. In cases of prolonged hospitalization without improvement in symptoms, parenteral nutrition may be indicated.

\section{Diclegis}

Diclegis has been the newly introduced resurgent therapy option for nausea and vomiting. Originally marketed as Bendectin, released by Merrell Dow in 1956 and subsequently removed from the market in 1980s due to litigation costs, the medication has been reapproved as Diclegis by the Food and Drug Administration (FDA) in 2013. Currently, Diclegis is the only FDAapproved medication of nausea and vomiting of pregnancy and is rated as category A for pregnancy. This medication is comprised of delayed-release formulation of $10 \mathrm{mg}$ doxylamine succinate and $10 \mathrm{mg}$ pyridoxine $\mathrm{HCl}$ [39]. Doxylamine succinate is an antihistamine ( $\mathrm{H} 1$ blocker), which is responsible for the drug's sedative and anti-emetic effects and $10 \mathrm{mg}$ pyridoxine $\mathrm{HCl}$ is a formulation of vitamin B6. Vitamin B6 is known to be utilized by the body in over 160 enzyme activities including metabolic processes of amino acids, nucleic acids, unsaturated fatty acids, carbohydrates, glycogen, neurotransmitters, and porphyrin, and therefore

Hyperemesis Gravidarum thought to be a beneficial supplement in patients with a depleted nutritional state [40]. The safety of the drug has been extensively analyzed prior to its rerelease in the United States, as it had been continuously offered in Canada and Europe. A systematic analysis of data from 12 cohort and 5 case-control studies totaling close to 200,000 patients calculated the overall summary odds ratio to be 1.01, indicating the absence of any increased risk attributable to drug. Due to the expense of the patented formulation, patients and physicians have attempted multiple off-brand alternatives including offlabel use of over-the-counter $12.5 \mathrm{mg}$ doxylamine (0.5 tablets of $25 \mathrm{mg}$ ) plus $10-25 \mathrm{mg}$ pyridoxine; however, due to the nondelayed formulation of these alternatives, the efficacy of treatment appears diminished. Most studies, cite the effectiveness of Diclegis in cases classified as "nausea and vomiting" of pregnancy to be between 70 and $80 \%$ [40]. To date, Diclegis has not been studied specifically in patients with hyperemesis gravidarum. However, a study by Maltepe and Koren [41] suggests that preconception treatment with Diclegis can decrease the rates of hyperemesis gravidarum.

\section{Promethazine}

Promethazine, a type of antidopaminergic therapy, has been utilized in patients with hyperemesis gravidarum. Promethazine has multiple mechanisms of actions including weak central nervous system blockade of dopamine and seratonin receptors, muscarinin-blocking effects as well as strong, long-lasting antihistamine action. The antihistamine-blocking properties can be attributed to the increase in reported side effects of sedation for patients receiving promethazine treatment vs. patients receiving metoclopramide. Promethazine is available in multiple formulations including injectable solution, suppositories, tablets, and syrup (6.25 $\mathrm{mg} / 5 \mathrm{~mL}$ ). While the preferred administration route is oral, in the case of hyperemesis gravidarum, rectal suppositories and intramuscular administration are acceptable alternatives. Intravascular administration is less recommended due to increased risk of local tissue damage at the site of administration [42]. Promethazine is utilized mainly as a second-stage agent in patients who are refractory to initial treatment. A randomized control study of 75 patients hospitalized for hyperemesis gravidarum did not demonstrate a difference in effectiveness between intravenous treatment with $25 \mathrm{mg}$ prometha- 
zine or $10 \mathrm{mg}$ metoclopramide every $8 \mathrm{~h}$ for $24 \mathrm{~h} \mathrm{[43].}$ As an initial therapeutic agent, a study of promethazine versus metoclopramide in combination with pyroxamine showed that the later treatment resulted in higher rates of reported improvement as stated by patients [44]. Promethazine has been shown to cross the placental barrier and be expressed in breast milk. It is considered to be a category $\mathrm{C}$ drug due to potential neurologic effects on the fetus [45].

\section{Clonidine}

Clonidine, a centrally acting alpha-agonist, is commonly utilized as an antihypertensive agent; however, it has also been referenced in treatment of patients with refractory hyperemesis gravidarum [27]. Clonidine is available in the tablet form as well as in a solution and a transdermal form. A recent pilot study (CLONEMESI trial) of 12 women with severe refractory hyperemesis gravidarum who were treated with $5 \mathrm{mg}$ transdermal clonidine patch for 5 days demonstrated a significant improvement in symptoms in the treatment group. Outcomes of this study showed a significant improvement in PUQE scores and (visual analog scale) scores, as well as a decrease in laboratory abnormalities such as electrolyte imbalances and ketonuria. In the future, a more powered study will be required to analyze the efficacy and safety of clonidine in hyperemesis gravidarum [46].

\section{Metoclopramide and Zofran}

Metoclopramide and Zofran are the mainstays of current hyperemesis gravidarum therapy. The complete mechanism of action of metoclopramide has not been elucidated; however, it is known to be an anti-HT3 medication and to have antidopaminergic properties. It is known to affect gastrointestinal tract peristalsis and CNS signaling, especially in the area of the medullary chemoreceptor trigger zone. Metoclopramide is used in a variety of settings including nasogastric tube placement, chemotherapy-induced nausea and vomiting, diabetic gastroparesis, severe GERD and postoperative nausea/vomiting, and ileus. It is available in multiple formulations including injections, syrup, and in the tablet form. Metoclopramide is supplied with a "black box" warning of possible irreversible tardive dyskinesia and is absolutely contraindicated in patients who are taking other medications with possible extrapyramidal effects. It may also cause worsening of preexisting psychiatric conditions and well as QT prolongation, among many other side effects. Metoclopramide should not be used for more than 12 weeks. It is currently rated as pregnancy category B agent [47]. A large cohort study of over 28,000 patients receiving Reglan showed no observed association between exposure to metoclopramide during pregnancy and an increased risk of birth defects, spontaneous abortion, or stillbirth [48].

The use of Zofran started as primarily an agent for chemotherapy-induced nausea/vomiting, as it has been shown to be superior to other antiemetics for this purpose. Zofran is also used in postoperative and postradiation nausea, as well as in nausea and vomiting of pregnancy. Unlike metoclopramide, Zofran does not promote gastrointestinal motility and is therefore an agent of choice in cases of emesis and diarrheal symptoms. Zofran is available in an oral or injectable form and may cause significant allergic reactions. This medication has also been associated with QT prolongation. Since it does not promote gastrointestinal motility, Zofran is not recommended in cases of nausea where gastroparesis or ileus may be a concern [49]. Zofran has been used for treatment of hyperemesis gravidarum since the 1990s; however, its utilization in the United States was briefly decreased when reports of its associations with fetal cardiac malformations were published by a Swedish study. Further analysis, including a large cohort of over 7,000 patients showed no significant association between Zofran use and fetal malformations [50, 51]. In recent years, Zofran is again becoming the most prescribed medication for primary treatment of patients with hyperemesis gravidarum [52]. A recent RCT did not show any differences in efficacy between metoclopramide or Zofran when therapy was randomly assigned to $160 \mathrm{pa}-$ tients with hyperemesis gravidarum [53]. In addition, meta-analysis of studies assessing intravenous pump infusions of metoclopramide and Zofran showed no benefit for continued use and in fact, showed an increased risk of side effects of this treatment modality, including extra-pyramedial symptoms and worsening of nausea/ vomiting [54].

\section{Mirtazapine}

The use of mirtazapine has been suggested in literature for treatment of patients with refractory hyperemesis gravidarum. Mirtazapine has been found to be specifi- 
cally beneficial in cases of hyperemesis gravidarum compounded by a psychiatric diagnosis such as depression [55]. Mirtazapine is in a class of medications called NaSSA: noradrenergic and specific serotonergic antidepressant, and acts by antagonizing adrenergic alpha2-receptors and by blocking serotonin 5-HT2 and 5-HT3 receptors. It also has histaminergic and muscarinic effects. It is believed that the anti-5HT3 action of the drug provides the anti-emetic properties, similar to that of Zofran, which also functions by antagonizing $5 \mathrm{HT} 3$ receptor. Antagonism of 5-HT2 and 5-HT3 receptors also contributes to the anxiolytic, sedative, and appetite-stimulating effects [52]. Administration of the drug at a standard daily dosing of $15 \mathrm{mg}$ has been shown to reach steady state in approximately 4 days [56]. In a case series review of 15 published cases of patients with hyperemesis gravidarum who have failed other forms of management, 8 patients were documented to have a response to mirtazapine treatment within one week [52]. According to some reports, the effect of treatment was so significant that it allowed patients to continue the pregnancy when they would have otherwise elected a termination. Mirtazapine is currently FDA approved for treatment of Major Depressive Disorder and has with off-label use in other psychiatric conditions including posttraumatic stress disorder, panic disorder, social anxiety disorder, obsessive-compulsive disorder, insomnia, somatoform disorder, and in schizophrenia.

A number of studies have evaluated mirtazapine and its safety in pregnancy. A meta-analysis assessed patients who were exposed to $30 \mathrm{mg}$ of mirtazapine versus exposure to selective serotonin reuptake inhibitors (SSRI) versus no exposure to any medical intervention. Primary outcomes measured were severe structural impairment and anomalies requiring surgical correction, and secondary outcomes were of birth complications such as rate of stillbirth, preterm deliveries, and birth weight. These analyses showed no statistically significant difference in major birth defects between mirtazapine and SSRI groups; however, a small overall increase in the percentages of major birth defects between the mirtazapine, SSRI, and the no-exposure group was noted $(4.1,4.2$, and $1.3 \%$, respectively, $p=0.07)$. When exposure was sub-analyzed to only those exposed in the first-trimester, no significant increased risk was noted between the mirtazapine, SSRI, or the no-intervention group. Another meta-analysis of 390 cases of neonates exposed to mirtazapine found no associated increased rates in major defects [57]. Limited data are available regarding mirtazapine and lactation; however, the same meta-analysis suggests that use of mirtazapine during breastfeeding is safe due to the relatively low infant dosage [58].

\section{Corticosteroids}

Evidence of the benefit of corticosteroid treatment of hyperemesis gravidarum remains controversial. In our review, only limited randomized clinical trials were assessed, mainly comparing corticosteroid treatment to placebo in addition to routine hospital management. One of the largest available studies, an RCT in 2003, assessed corticosteroid supplementation in 56 patients to the routine treatment regimen of metoclopramide and promethazine. The primary outcome of the study was rehospitalization for hyperemesis, which occurred in $34 \%$ of women who received corticosteroids compared with $35 \%$ in women who received placebo [59]. In another study, patients hospitalized in intensive care units for hyperemesis gravidarum were treated with a dose of either intravenous hydrocortisone 300 or $10 \mathrm{mg}$ intravenous metoclopramide 3 times daily. The outcome analysis of the study showed a significant reduction in the number of emesis episodes starting from the second day of treatment in the hydrocortisone group [60]. The safety of corticosteroids, mainly prednisone, has been debated. Prednisone is currently rated as a category $\mathrm{C}$ drug. Meta-analysis of exposure to prednisone during the first trimester of pregnancy revealed a positive association between fetal oral cleft defects and prednisone use [61].

Therapeutic options in the treatment of patients with hyperemesis gravidarum are outlined in Table 1.

\section{Summary}

Hyperemesis gravidarum remains a disease of diagnosis based on clinical judgment and regretfully not upon strict, well-defined diagnostic criteria. Variability in the clinical definition contributes to the difficulty in performing meta-analyses of available research and currently hyperemesis gravidarum does not have a clearly established etiology. New research in the field, however, appears to be promising especially in the realm of genetics and familial predisposition to development of hyperemesis gravidarum. Although current literature appears to be in agreement with maternal morbidity, studies disagree on the association of fetal morbidity and hyper- 
Table 1. Therapeutic options of hyperemesis gravidarum

\begin{tabular}{|c|c|c|c|}
\hline Drug & Mechanism & Clinical benefit & Safety \\
\hline Diclegis & $\begin{array}{l}\text { Antihistamine (H1 blocker) with vitamin } \\
\text { B6 (cofactor in enzymatic reactions) }\end{array}$ & $\begin{array}{l}\text { Mainly in nausea/vomiting of pregnancy. } \\
\text { May aid in pretreatment of HG }\end{array}$ & $\begin{array}{l}\text { FDA approved for N/V } \\
\text { of pregnancy } \\
\text { Pregnancy category A }\end{array}$ \\
\hline Ondansetron & Selective 5-HT3 receptor antagonist & First-line treatment in $\mathrm{HG}$ & Pregnancy category B \\
\hline Metoclopramide & $\begin{array}{l}\text { Anti-HT3 antidopaminergic properties. } \\
\text { Prokinetic agent }\end{array}$ & First-line treatment in $\mathrm{HG}$ & Pregnancy category B \\
\hline Clonidine & Centrally acting alpha-agonist & Pilot studies show benefit in refractory HG & Pregnancy category C \\
\hline Promethazine & $\begin{array}{l}\text { Weak antidopaminergic and antiserotonin } \\
\text { receptor activity in CNS, antimuscarinic, } \\
\text { long-lasting antihistamine action }\end{array}$ & $\begin{array}{l}\text { Small-scale studies for refractory HG } \\
\text { show benefit of addition of promethazine to } \\
\text { first-line treatment }\end{array}$ & Pregnancy category C \\
\hline Prednisone & Multifactorial & $\begin{array}{l}\text { Small-scale studies show addition of } \\
\text { prednisone decreases daily episodes of emesis }\end{array}$ & Pregnancy category C \\
\hline Mirtazapine & $\begin{array}{l}\text { Multifactorial: adrenergic alpha2 } \\
\text { antagonist, serotonin 5-HT2 and 5-HT3 } \\
\text { antagonism with histaminergic and } \\
\text { muscarinic effects }\end{array}$ & $\begin{array}{l}\text { Shows benefit in symptomatic relief in } \\
\text { small-scale studies of refractory HG }\end{array}$ & $\begin{array}{l}\text { Pregnancy category C } \\
\text { Strong association of } \\
\text { prednisone exposure and } \\
\text { fetal oral cleft defects }\end{array}$ \\
\hline
\end{tabular}

emesis gravidarum. The main focus of disagreement is the effect of hyperemesis gravidarum on gestational age at delivery and birth weight. The bulk of available research in the area is focused on therapeutic options and studies of multiple first-line agents including on- dansetron and metoclopramide, which appear to show similar efficacy and safety. Studies on therapeutics for refractory hyperemesis gravidarum are few in number, suggesting opportunities for further research in this arena.

\section{References}

1 Daniels J: Hyperemersis gravidarum: past hysteria and present needs. BJOG 2017;124:31.

2 Bacon CS: The vomiting of pregnancy. Am J Med Sci 1898;115:680-683.

3 Ping E, Virginia W: Causes and treatment modalities of Hyperemesis Gravidarum during the late nineteenth and early twentieth century in the United States and Great Britain. American Public University, 2010.

4 American College of Obstetricians and Gynecologists: Practice Bulletin No. 153: nausea and vomiting of pregnancy. Obstet Gynecol 2015;126:e12-e24.

5 Boelig RC, Barton SJ, Saccone G, Kelly AJ, Edwards SJ, Berghella V: Interventions for treating hyperemesis gravidarum. Cochrane Database Syst Rev 2016;5:1-147.

6 Fejzo MS, Ingles SA, Wilson M, Wang W, MacGibbon K, Romero R, Goodwin TM: High prevalence of severe nausea and vomiting of pregnancy and hyperemesis gravidarum among relatives of affected individuals. Eur J Obstet Gynecol Reprod Biol 2008;141: 13-17.
7 Patterson AM: Hyperemesis Gravidarum. Ferri's Clinical Advisor 2017;6:629-629i.

8 Lee NM, Saha S: Nausea and vomiting of pregnancy. Gastroenterol Clin North Am 2011;40:309-334.

9 Ebrahimi N, Maltepe C, Bournissen FG, Koren G: Nausea and vomiting of pregnancy: using the 24-hour pregnancy-unique quantification of emesis (PUQE-24) scale. J Obstet Gynaecol Can 2009;31:803-807.

10 Loh KY, Sivalingam N: Understanding hyperemesis gravidarum. Med J Malaysia 2005;60: 394-399.

11 Eliakim R, Abulafia O, Sherer DM: Hyperemesis gravidarum: a current review. Am J Perinatol 2000;17:207-218.

12 Niemeijer MN, Grooten IJ, Vos N, Bais JM, van der Post JA, Mol BW, Roseboom TJ, Leeflang NM, Painter RC: Diagnostic markers for hyperemesis gravidarum: a systematic review and metaanalysis. Am J Obstet Gynecol 2014;211:150.e1e15.

13 Kjeldgaard HK, Eberhard-Gran M, Benth JŠ, Nordeng H, Vikanes ÅV: History of de- pression and risk of hyperemesis gravidarum: a population-based cohort study. Arch Womens Ment Health 2017;20:397404.

14 Christodoulou-Smith J, Gold JI, Romero R, Goodwin TM, Macgibbon KW, Mullin PM, Fejzo MS: Posttraumatic stress symptoms following pregnancy complicated by hyperemesis gravidarum. J Matern Fetal Neonatal Med 2011;24:1307-1311.

15 Verberg MF, Gillott DJ, Al-Fardan N, Grudzinskas JG: Hyperemesis gravidarum, a literature review. Hum Reprod Update 2005;11: 527-539.

16 Grooten IJ, Den Hollander WJ, Roseboom TJ, Kuipers EJ, Jaddoe VW, Gaillard R, Painter RC: Helicobacter pylori infection: a predictor of vomiting severity in pregnancy and adverse birth outcome. Am J Obstet Gynecol 2017;216: 512.e1-e9.

17 Li L, Li L, Zhou X, Xiao S, Gu H, Zhang G: Helicobacter pylori infection is associated with an increased risk of hyperemesis gravidarum: a meta-analysis. Gastroenterol Res Pract 2015;2015:278905. 
18 Golberg D, Szilagyi A, Graves L: Hyperemesis gravidarum and Helicobacter pylori infection: a systematic review. Obstet Gynecol 2007; 110:695-703.

19 Selitsky T, Chandra P, Schiavello HJ: Wernicke's encephalopathy with hyperemesis and ketoacidosis. Obstet Gynecol 2006;107:486490.

20 Chiossi G, Neri I, Cavazzuti M, Basso G, Facchinetti F: Hyperemesis gravidarum complicated by Wernicke encephalopathy: background, case report, and review of the literature. Obstet Gynecol Surv 2006;61:255-268.

21 Giugale LE, Young OM, Streitman DC: Iatrogenic Wernicke encephalopathy in a patient with severe hyperemesis gravidarum. Obstet Gynecol 2015;125:1150-1152.

22 Sutamnartpong $\mathrm{P}$, Muengtaweepongsa S, Kulkantrakorn K: Wernicke's encephalopathy and central pontine myelinolysis in hyperemesis gravidarum. J Neurosci Rural Pract 2013;4:39-41.

23 Baba Y, Morisawa H, Saito K, Takahashi $\mathrm{H}$, Rifu K, Matsubara S: Intraperitoneal hemorrhage in a pregnant woman with hyperemesis gravidarum: vitamin $\mathrm{K}$ deficiency as a possible cause. Case Rep Obstet Gynecol 2016; 2016:5384943.

24 Lassey SC, Robinson JN: Rhabdomyolysis after hyperemesis gravidarum. Obstet Gynecol 2016;128:195-196.

25 Buchanan GM, Franklin V: Hamman and Boerhaave syndromes - diagnostic dilemmas in a patient presenting with hyperemesis gravidarum: a case report. Scott Med J 2014; 59:e12-e16.

26 Poursharif B, Korst LM, MacGibbon KW, Fejzo MS, Romero R, Goodwin TM: Elective pregnancy termination in a large cohort of women with hyperemesis gravidarum. Contraception 2007;76:451-455.

27 Simpson SW, Goodwin TM, Robins SB, Rizzo AA, Howes RA, Buckwalter DK, Buckwalter JG: Psychological factors and hyperemesis gravidarum. J Wones Health Gend Based Med 2001;10:471-477.

28 Hizli D, Kamalak Z, Kosus A, Kosus N, Akkurt G: Hyperemesis gravidarum and depression in pregnancy: is there an association? J Psychosom Obstet Gynaecol 2012;33:171-175.

29 Tan PC, Zaidi SN, Azmi N, Omar SZ, Khong SY: Depression, anxiety, stress and hyperemesis gravidarum: temporal and case controlled correlates. PLoS One 2014 9:e92036.

30 Tan PC, Vani S, Lim BK, Omar SZ: Anxiety and depression in hyperemesis gravidarum: prevalence, risk factors and correlation with clinical severity. Eur J Obstet Gynecol Reprod Biol 2010;149:153-158.

31 Saha S, Loranger D, Pricolo V, Degli-Esposti S: Feeding jejunostomy for the treatment of severe hyperemesis gravidarum: a case series. JPEN J Parenter Enteral Nutr 2009;33:529_ 534

32 Dodds L, Fell DB, Joseph KS, Allen VM, Butler B: Outcomes of pregnancies complicated by hyperemesis gravidarum. Obstet Gynecol 2006; 107:285-292.

33 Kuru O, Sen S, Akbayır O, Goksedef BP, Ozsürmeli M, Attar E, Saygilı H: Outcomes of pregnancies complicated by hyperemesis gravidarum. Arch Gynecol Obstet 2012;285: 1517-1521.

34 Koudijs HM, Savitri AI, Browne JL, Amelia D, Baharuddin M, Grobbee DE, Uiterwaal CS: Hyperemesis gravidarum and placental dysfunction disorders. BMC Pregnancy Childbirth 2016;16:374.

35 Bolin M, Akerud H, Cnattingius S, Stephansson $\mathrm{O}$, Wikström AK: Hyperemesis gravidarum and risks of placental dysfunction disorders: a population-based cohort study. BJOG 2013;120:541-547.

36 Mullin PM, Bray A, Schoenberg F, MacGibbon KW, Romero R, Goodwin TM, Fejzo MS: Prenatal exposure to hyperemesis gravidarum linked to increased risk of psychological and behavioral disorders in adulthood. J Dev Orig Health Dis 2011;2:200-204.

37 Tan P, Norazilah MJ, Omar SZ: Dextrose saline compared with normal saline rehydration of hyperemesis gravidarum: a randomized controlled trial. Obstet Gynecol 2013; 121(2 pt 1):291-298.

38 Spruill SC, Kuller JA: Hyperemesis gravidarum complicated by Wernicke's encephalopathy. Obstet Gynecol 2002;99(5 pt 2):875-877.

39 Longo L: Delayed-Release Doxylamine-Pyridoxine Drug Monograph. VA Pharmacy Benefits Management, 2015.

40 Madjunkova S, Maltepe C, Koren G: The delayed-release combination of doxylamine and pyridoxine $\left(\right.$ diclegis $^{\circledR} /$ diclectin $^{\circledR}$ ) for the treatment of nausea and vomiting of pregnancy. Pediatr Drugs 2014;16:199-211.

41 Maltepe C, Koren G: Preemptive treatment of nausea and vomiting of pregnancy: results of a randomized controlled trial. Obstet Gynecol Int 2013;2013:1-8.

42 Smith HS, Cox LR, Smith BR: Dopamine receptor antagonists. Ann Palliat Med 2012;1: 137-142.

43 Tan PC, Khine PP, Vallikkannu N, Omar SZ: Promethazine compared with metoclopramide for hyperemesis gravidarum: a randomized controlled trial. Obstet Gynecol 2010;115:975-981

44 Bsat FA, Hoffman DE, Seubert DE: Comparison of three outpatient regimens in the management of nausea and vomiting in pregnancy. J Perinatol 2003;23:531-535.

45 Sanofi-Aventis: Phenergan Product Information, 2012.

46 Maina A, Arrotta M, Cicogna L, Donvito V, Mischinelli M, Todros T, Rivolo S: Transdermal clonidine in the treatment of severe hyperemesis. A pilot randomised control trial: CLONEMESI. BJOG 2014;121:1556-1562.

47 American Society of Health - System Pharmacists, Metoclopramide Hydrochloride Drug Monograph. AHFS DI Essentials, 2017.

48 Pasternak B, Svanström H, Mølgaard-Nielsen D, Melbye M, Hviid A: Metoclopramide in pregnancy and risk of major congenital malformations and fetal death. JAMA 2013;310: 1601-1611.

49 American Society of Health - System Pharmacists, Zofran Drug Monograph. AHFS DI Essentials, 2017.

50 Pasternak B, Svanstrom H, Hviid A: Ondansetron in pregnancy and risk of adverse fetal outcomes. N Engl J Med 2013;368:814-823.

51 Einarson A, Maltepe C, Navioz Y, Kennedy D, Tan MP, Koren G: The safety of ondansetron for nausea and vomiting of pregnancy: a prospective comparative study. BJOG 2004;111: 940-943.

52 Abramowitz A, Miller ES, Wisner KL: Treatment options for hyperemesis gravidarum. Arch Womens Ment Health 2017;20:363372

53 Abas MN, Tan PC, Azmi N, Omar SZ: Ondansetron compared with metoclopramide for hyperemesis gravidarum: a randomimzed controlled trial. Obstet Gynecol 2014;123: 1272-1279.

54 Reichmann JP, Kirkbride MS: Reviewing the evidence for using continuous subcutaneous metoclopramide and ondansetron to treat nausea \& vomiting during pregnancy. Manag Care 2012;21:44-47.

55 Omay O, Einarson A: Is mirtazapine an effective treatment for nausea and vomiting of pregnancy?: a case series. J Clin Psychopharmacol 2017;37:260-261.

56 Anttila S, Leinonen EV: A review of the pharmacologicaland clinical profile of mirtazapine. CNS Drug Rev 2001;7:249-264.

57 Winterfeld U, Klinger G, Panchaud A, Stephens S, Arnon J, Malm H, Te Winkel B, Clementi $\mathrm{M}$, Pistelli $\mathrm{A}$, Maňáková E, Eleftheriou G, Merlob P, Kaplan YC, Buclin T, Rothuizen LE: Pregnancy outcome following maternal exposure to mirtazapine: a multicenter, prospective study. J Clin Psychopharmacol 2015;35:250 259.

58 Smit M, Dolman KM, Honig A: Mirtazapine in pregnancy and lactation - a systematic review. Eur Neuropsychopharmacol 2016;26: 126-135.

59 Yost NP, McIntire DD, Wians FH Jr, Ramin SM, Balko JA, Leveno KJ: A randomized, placebo-controlled trial of corticosteroids for hyperemesis due to pregnancy. Obstet Gynecol 2003;102:1250-1254.

60 Bondok RS, El Sharnouby NM, Eid HE, Abd Elmaksoud AM: Pulsed steroid therapy is an effective treatment for intractable hyperemesis gravidarum. Crit Care Med 2006;34:27812783.

61 Park-Wyllie L, Mazzotta P, Pastuszak A, Moretti ME, Beique L, Hunnisett L, Friesen $\mathrm{MH}$, Jacobson S, Kasapinovic S, Chang D, Diav-Citrin O, Chitayat D, Nulman I, Einarson TR, Koren G: Birth defects after maternal exposure to corticosteroids: prospective cohort study and meta-analysis of epidemiological studies. Teratology 2000; 62:385-392. 